\title{
Various Segmentation Techniques for Lung Cancer Detection using CT Images: A Review
}

\author{
J. Vijayaraja ${ }^{a}$ and D. Loganathan ${ }^{b}$
}

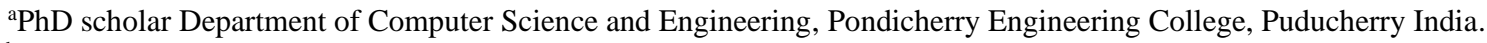
bProfessor Department of Computer Science and Engineering, Pondicherry Engineering College, Puducherry India.

Article History: Received: 11 January 2021; Accepted: 27 February 2021; Published online: 5 April 2021

\begin{abstract}
Computed Tomography (CT) is far and wide utilized to make a diagnosis and access thoracic diseases. The enhanced resolution of CT examination has resulted in a considerable investigation of statistics for analysis. Computerizing the scrutiny of such facts is consequently necessitate and fashioned a hastily emergent research region in medical imaging. The finding of thoracic diseases by means of image processing directs to a pre- processing step identified as "Lung segmentation" which portrays a wide range of techniques starts with simple Thresholding and numerous image processing elements are incorporated to progress segmentation, precision and heftiness. In image processing, techniques like image pre-processing, segmentation and feature extraction have been thrashed out in detail. This paper suggestions investigation of literature on computer examination of the lungs in CT scans and statements the Preprocessing ideas, segmentation of a choice of pulmonary arrangements, and Feature Extraction intended at recognition and categorization of chest abnormalities. As well as, research developments and disputes are recognized and instructions for further examinations are discussed.
\end{abstract}

Keywords: CT, Segmentation, Thresholding, Preprocessing, Feature Extraction.

\section{Introduction}

Computer Tomography (CT) is extensively cast-off by clinical radiologist for the rationale of identifying and treating thoracic diseases. Computed tomography (CT) is an imaging test method utilized to generate diagnose comprehensive images of internal organs, soft tissues, bones and blood vessels (Khin, 2014; Song, 2012; Bhasa, 2020)]. CT scan cross-sectional images can be reformatted in a variety of planes to create three-dimensional images that can be seen on a screen display, printed on paper, or transferred to electronic media. CT scanning is frequently the finest method for sensing a lot of diverse cancers as the images permit the doctor to substantiate the occurrence of a tumor and find out its size and location (Wei, 2014). CT is rapid, effortless, non-invasive and precise. In urgent situations, it can disclose internal injuries and flow of blood rapidly sufficient to assist save lives. Since it is capable to sense very small nodules in the lung, chest CT is particularly effective for detecting lung cancer at its most primitive, most curable period. CT images are digitized (Sluimer, 2006; Deepthi, 2019; Chinnamahammad bhasha, 2020) for enhancing the image reconstruction modus operandi and to supplement the image analysis techniques. The preliminary step in image examination is "Segmentation", where the organ (lungs) is perceived and anatomic precincts are demarcated either manually or automatically (Amutha, 2012; Balamurugan, 2020). The succeeding step is to reduce the false positive outcomes in the segmentation area and to improve the accuracy on performing segmentation. Image segmentation in this high- to low-level chain of command is an amalgamation of recognition and delineation is thus illustrated with a pulmonary CT (Rikxoort, 2013; ChinnamahammadBhasha, 2020) image shown in fig 1. The former recognition step is carried out by recognizing the left and right lung field by user interaction, the latter is user-provided information is processed to situate the precise boundary of the lung fields.
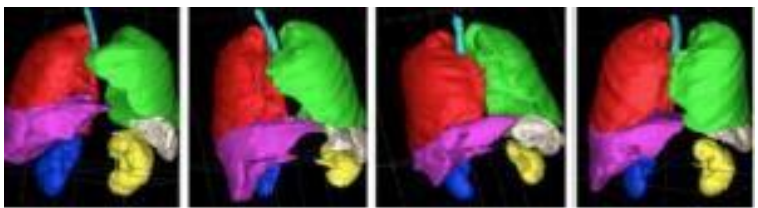

Figure 1. Image Segmentation of Lung

Likewise, the pneumothorax occurrence or pleural effusion (Mansoor , 2014; ChinnamahammadBhasha, 2020) on a CT image can extensively disfigure the domino consequence of computerized segmentation, hence leading to erroneous quantification. 


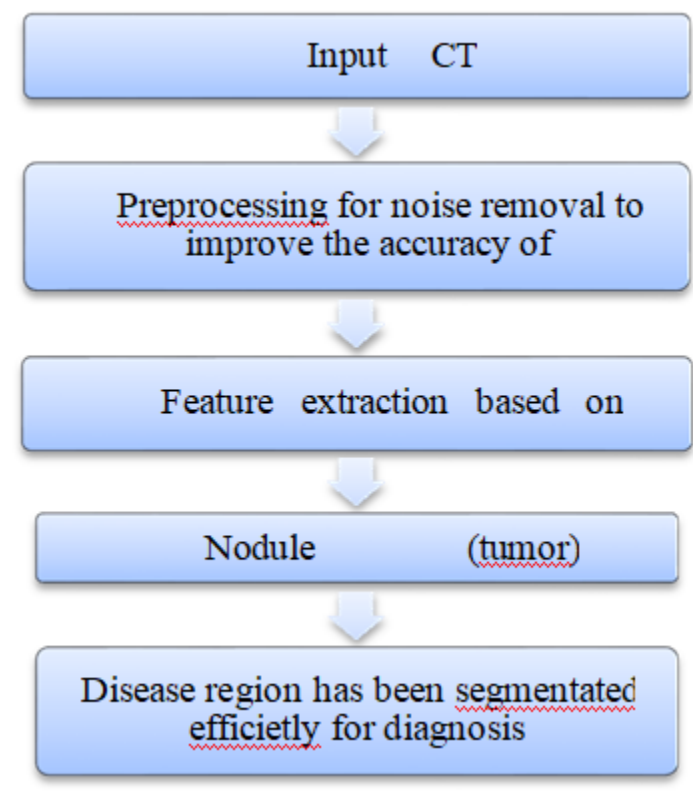

Figure 2. Flow Chart for Segmentation technique

The overall process of CT image segmentation based on image processing schemes is shown in fig 2 . The main aid of segmentation is to split the whole region into small regions. Based on this, the disease affected regions are separated from whole image region for efficient disease diagnosis. Initially, the CT lungs input image is preprocessed to reduce impulse noise and to improve image quality for improving the segmentation accuracy. Then, the lung regions are segmented to reduce the processing time. After that, the some features are extracted for segmentation analysis. Finally, five types of segmentation schemes are processed and segmented the disease (i.e. tumor) affected regions.

Organization of this paper is as follows. In Section II, pre-processing stages are introduced. In Section III, the diverse segmentation techniques are proposed to enhance the accurateness. Feature Extraction is shown in Section IV. Problem Specifications and future investigational ideas are elaborated in Section V trailed by conclusion in section VI.

\section{Preprocessing}

To begin with, the image was transformed to gray scale image that includes only brightness data. These images are resolute as a 2-D array of pixels by means of 8 bits/pixels. At this juncture, a pixel value of 0 is black and value of 255 is white, with intermediate values consequent to unreliable shades of gray. Benefit of transferring an image to gray scale is to diminish the processing time.

The spatial domain and frequency domain are the two general categories of image enhancement approaches (ChinnamahammadBhasha, 2020; Garikapati P, 2020). The spatial domain encompasses direct image pixel manipulation, while the frequency domain encompasses the manipulation of an image's Fourier transform or wavelet transform interpretability or sensitivity of data embedded in them to provide improved feedback for other programmed image processing techniques. To increase image quality, the RGB image is first transformed to a grey scale image, as seen in fig 3. Following that, image recognition schemes are used.

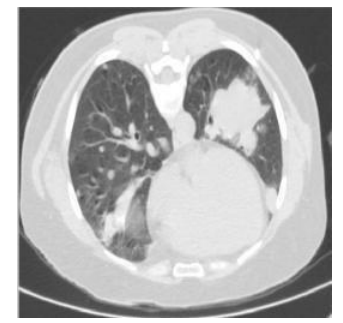

a) Original Image

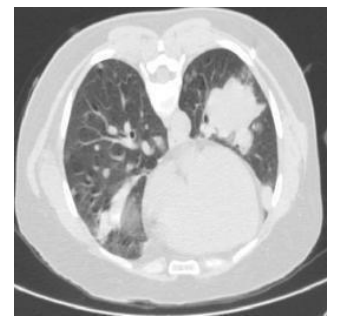

b) Gray Scale Image

Figure 3. Image processing results 
Conversely, when considering image enhancement methods as preprocessing tools for other image processing techniques, the proven measures can establish which modus operandi are most suitable.

\subsection{Histogram Equalization}

Histogram Equalization is utilized in image enhancement method. Pre-processing of image endeavors for removal of redundancy in scanned images devoid of disturbing novel image, which takes a vital role in scrutiny of lung cancer. As a upshot, Histogram- Equalization turns out to be the essential step in preprocessing. Therefore, every single image is preprocessed to improve its superiority.

\subsection{Noise Removal}

In medical image processing, it is extremely significant to attain accurate images to support precise elucidation for the provided application (Aroulanandam, 2020). Low image quality is an obstruction for effectual feature extraction, analysis, identification and quantitative measurements. For this reason, there is a crucial demand of noise diminution from medical images. The noise filtered image is displayed in fig 4. There are huge sums of imaging modalities that are cast off for the investigation of medical image processing.

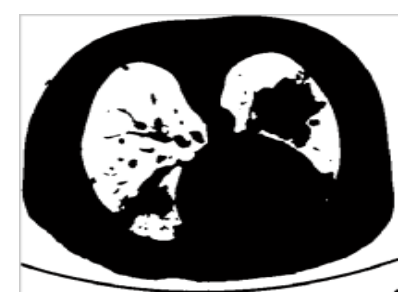

Figure 4. Filtered Image

\subsubsection{MRI}

The magnetic resonance imaging (MRI) is a common diagnostic tool (Hari, 2014; Balamurugan, 2017; Balamurugan, 2018). Preprocessing removes noise and other irregularities from the image while still sharpening the edges. This is also where RGB to grey conversion and reshaping takes place. It has a median filter for noise reduction. The likelihood of noise influx in a current CT scan is very low. It could show up as a result of the thermal outcome.

\subsubsection{Median Filtering Median filtering}

The median filtering technique removes noise from images in a nonlinear way. It's popular because it's good at removing noise while keeping the edges in tact. It's particularly effective at removing sounds like "salt and pepper." By stirring through the image pixel by pixel, the median filter restores each value to the median value of neighboring pixels (Latchoumi, 2020; Pavan, 2020). The "glass" is the outline of neighbors, which slides pixel by pixel instead of the whole image pixel by pixel. The median is determined by first cataloging all of the pixel values from the window into arithmetical order, and then restoring the pixel being measured with the middle (median) pixel value.

\section{Feature Extraction}

Function extraction is one of most critical activities. Following segmentation, next step is feature extraction, which is performed on segmented lung regions obtained in previous step to distinguish one area of interest from another.

Feature Extraction from Segmented Region subsequent to the segmentation is executed on lung region, characteristics can be gained from it for determining diagnosis rule for identifying cancer nodules in lung region completely. Features (Khin , 2014)that are cast off in this work in order to generate diagnosis rules are:

1) Area: The region can be achieved by shortening pixel areas in the image recorded in the binary image (Yao, 2013). $A=n\{1\}$ where, $n\{\}$ the number of the curly brackets of the pattern sums.

2) Perimeter: The pixel number in the object's boundary is the perimeter [length]. Perimeter $P$ is intended to summarize the distances between any consecutive limit point (Chen, 2012).

3) Eccentricity: The excentricity is the relation between the length of its foremost axis and the distance between the ellipse's focal points. It ranges from 0 to 1 . Using the Gray-Level Matrix (GLCM), which is one of the most commonly used methods for texture processing, a texture attribute extraction is carried out on a quantified image after physical dimension determination. A second order of statistical measure, 
Haralick applied a co-occurrence gray level matrix.

4) Entropy: Determination of statistical randomness to determine the structure of the input image. Entropy $=-$ where $\mathrm{p}$ is GLCM's co-occurrence number of gray-level matrices.

5) Contrast: Measures spatial changes of the GLCM. The intensity difference between a pixel and its neighbors is calculated for the whole scene. For a constant image, dissimilarity is 0 . (i- j) $\sum \sum$ Contrast $=2$ $\mathrm{p}(\mathrm{i}, \mathrm{j})(12)$ Where, $\mathrm{P}(\mathrm{i}, \mathrm{j})=$ pixel at location $(\mathrm{i}, \mathrm{j})$

6) Correlation: The joint frequency occurrence of the same pixel pairs is calculated.

7) Energy: In the GLCM, it provides the number of squared rudiments. It's also known as the angular second moment or uniformity. $(p(i, j)) 2(14) \sum \sum$ Energy=

8) Homogeneity: The distance between the GLCM diagonal and the distribution of components in the GLCM.

\section{Segmentation}

\subsection{Threshold Based Image Segmentation:}

The process of lung segmentation known as threshold dependent image segmentation is a powerful one. It's a fully automated system that combines an improved grey-level Thresholding algorithm with a refinement process. Traditional Thresholding strategies have a hard time choosing the right threshold. We build up an improved Thresholding method that does not necessitate choosing any factors and is robust on diverse CT images. Fig 5 reveals the Image segmentation based on Threshold technique.

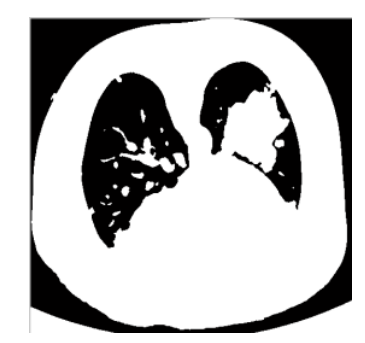

Figure 5. Threshold Image

Thresholding grounded segmentation, on the other hand, also has a crisis on the boundary wall and the regions of warships, which cause gaps in infinite segmentation borders. We develop a refinement method based on a texture-aware active contour model to address this issue. The texture of vessels, intensity properties, and structural features of the lungs are all included in this model. A satisfying segmentation is created by optimizing the replica to achieve both area and boundary consistencies. The segmentation is a lot more accurate this time. Noises and gaps are separated from the boundary, which is connected with true lung boundaries.

\subsection{Spiculation Segmentation}

Spiral Scan Technology was used to create a two-dimensional image from three-dimensional data. For detailed nodule segmentation, the dynamic programming technique was used. The spiculation in the speculation (Chen, 2012) was not segmented using the above procedure, and it was located in an annular area beyond the nodule boundary. The widen area where spiculation can exist was created by increasing the persuaded range outward along the nodule boundary, based on specific nodule segmentation.

\subsection{Segmentation with HMMF model}

A novel method was proposed for emphysema quantification grounded on a segmentation of lung tissue by means of a Hidden Markov Measure Field (HMMF) model. This method has two advantages compared to prevailing approaches: 1) the appearance replica adjust to the image information offering heftiness with respect to variability in intensity distributions, and 2) the Markov field implements spatial coherence of the segmented regions(Sunil Kumar, 2014) on condition that strength with respect to noise. The anticipated segmentation not only generates forceful measures of EI, but also generates robust delineations of unhealthy region which can be sensible in significant subtypes of emphysema. The HMMF replica has been used for liver tumor segmentation. 


\subsection{D Fuzzy Fisher method}

At first, the 2D Fischer can easily see prominent features inside an image as a Rayleigh entropy. 2) Subsequently adding a fluid rule would reduce the chance of over-segmentation for non-target signal videos. 3) Finally, because 2D Fisher space variances point out an edge of an image, the increased 2D furrowing probabilities correspond to an increase in 2D furrowed borders (Hashemi, 2013). In addition, 2D fuzzy Fisher grounded integral image should be recognized to assist a variety of optimal computing methods in deciding the best threshold pair. This strategy is effective in reducing unnecessary calculations since it essentially creates a lookup table norm. As a consequence, the 2D fuzzy Fisher-based integral image has a unified fuzzy border that is easily recognizable. In the narrow strip of a 2D histogram, the quantum-based particle swarm optimizer (QPSO) is used for rapid calculation as the predetermined particles build up in a 2D narrow strip field.

\subsection{Multi-band watershed Segmentation}

Segmenting of the watershed increases the gray scale image value of each pixel, which enables the entire image to become a topographical relief. When selecting images, watershed lines will demarcate geo-object frontiers and enable the separation of the entire image into parts. Because the gradient crest lines (ie local maxima) match the geo-objects margins, a gradient picture is commonly used for transformation of the watershed (Mesanovic, 2011). Most studies use a panchromatic or a single band of multi-spectral images to create gradient images. The multi-band watershed segmentation method is expected to create rudimentary segments for further area merging as a complete advantage of all spectral knowledge for edge identification. The conversion of original image into segmented image using watershed method is shown in fig 6.

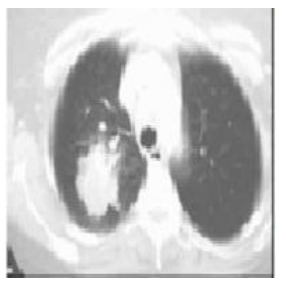

a) Original Image

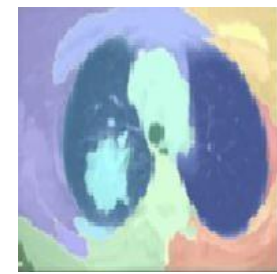

b) Segmented Image

Figure 6. Multi-band watershed Segmentation result

\subsection{Semi-automated segmentation of CT images}

Coronal CT slices contours (Lo, 2010) were obtained with a rudimentary algorithm approach that incorporated basic thresholds and morphological functions (Diciotti, 2011), and were considered to be suitable to detach arteries and new heart structures by the competent observer. Two sets of meshes, one for the left lung and the other for the right lung, were created. The form demonstration of the lung in CT images thus resulted in a surface mesh segment containing a series of points spread around the lung perimeter for each piece of image control.

\subsubsection{Vessel segmentation method:}

The proposed method extracts features using Gaussian pyramids [16] and a sparse auto-encoder, then instructs a random forest with these features and the ground reality. The Gaussian pyramid is used to obtain multiscale representations of pictures at first. The vessel detection modus operandi (Lassen, 2013) has been sprouting over a time by means of diverse techniques. Nevertheless, correct detection in advance stages leftovers a challenge. The research comprises for the progress of a vessel segmentation method through which shape and curvature of lungs can be recognized and airway elimination can be achieved.

\subsubsection{Pixel Machine Learning Pixel machine learning (PML)}

The traditional pixel-based image classification methodology (Nunes, 2010) classifies all pixels in an image pixel by pixel into land cover groups or themes. Figure 7 depicts the multispectral image transfer. Typically, multispectral data is used, with the spectral pattern found within the data for each pixel serving as the numerical basis for categorization. The pixel distance neighbor, parallelepiped, and utmost probability classifiers are the foundations for classical pixels.Methods of segmentation based on classification are supervised techniques. They demand a stage of preparation in which the training data is physically segmented. The evaluation data is repeatedly segmented as a result of the training segment outcome. There are several grouping methods demonstrated. Nonparametric classifiers and parametric classifiers are two types of classifiers. With respect to the nearest-neighbor classifier, the pixels fit in to the analysis information that is confidential in the similar class. 


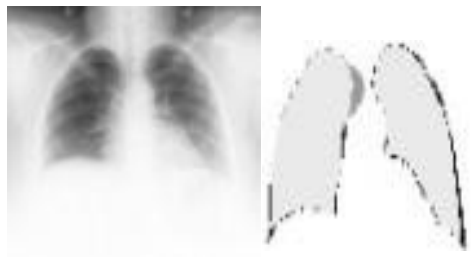

a) Original Image $\quad$ b) Pixel classified Image

Figure 7. Classification-based segmentation result

KNN is a systematic classification of the closest neighbor. Having regard to the weighted majority of votes cast by his neighbor, each pixel is classified as the most suitable class of its nearest $\mathrm{k}$ neighbours. In spatial simulation, the inconvenience of classification algorithms is deficient. This crisis is then lifted by the need to segment photographs that are diminished by severity. The accuracy of this algorithm depends in particular on the samples chosen for preparation.

\section{a. Neighboring segmentation}

There are three energy terms: the Potts smoothing term, the data term, and the probabilistic atlas term in medical image segmentation. A new possible feature that extends the data term is introduced in this paper. Where diverse objects have identical or similar CT values, the discriminating character of prevalent content, which was based on how specific the objects of interest materialize on the CT volume, has crisis. Find the CT values of a pair of adjacent voxels to triumph over this constraint.

To evaluate the increasing voxel of interest evaluated, the data term turn out to be added discriminable even if several objects of interest have similar CT values. The anticipated neighboring information term can be regarded as to unite the standard data term and the probabilistic atlas. CT volume is a group of tomographic images examined by means of X-ray and exploited to fabricate a 3D image of internal objects. As diverse objects blocks $\mathrm{X}$-ray beam in a different way, their CT values are observed in another way. A CT volume comprises of a group of voxels $\mathrm{V}$ and every single voxel $\mathrm{v} \in \mathrm{V}$ has its $\mathrm{CT}$ value Iv. The job of CT volume segmentation is to decay an input CT volume into a cluster of important objects, e.g., internal organs. It can be delight as a labeling crisis in which a label on behalf of an internal organ is allocated to every voxel. Let L be the set of target objects1; then the $\mathrm{CT}$ volume segmentation $\mathrm{L}$ allocates a label $1=\mathrm{Lv} \in \mathrm{L}$ to every voxel $\mathrm{v} \in \mathrm{V}$. The crisis of choosing the optimal labeling can be originated as an energy minimization crisis.

\section{b. 3D Histogram Technique:}

This approach counts the number of knowledge occurrences in a column in user-defined bins. A twodimensional graph, defined as a two-dimensional histogram, is made up of the sum of instances vs. bin number or vector value. Similarly, a three-dimensional histogram computes the number of knowledge occurrences from two columns in a grid. For lung filed segmentation (Nunes, 2010), 3D Histogram Thresholding is used, and devoid refinement is done using an SVM classifier. The characteristics are then extracted using 3D Co-occurrence matrices, and unnecessary features (Yrj, 2013) are omitted using step-wise discriminate analysis. The final move involves using a K-NN classifier to divide the characteristics into stable, interstitial pneumonia, and other lung disease trends. The step-wise discriminant investigation was used to select the Characteristics, and the cooccurrence matrices were used to extract attributes. The K-NN classifier was used to classify the features into stable, interstitial pneumonia, and other lung-diseased patterns in the final step (Chu,2013). When compared to fuzzy segmentation, the 3DHistogram Thresholding and Border Refinement performed is well-organized. The 3D histogram method's input and output images are seen in Fig. 8.

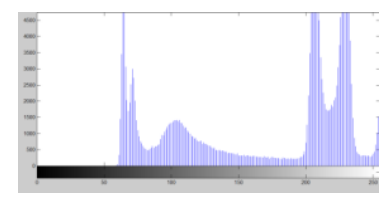

a) Histogram of Original Image

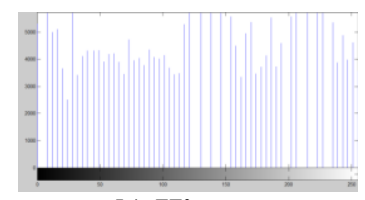

b) Histogram Equalization

Figure 8. Histogram result

\subsection{Deformable Model Method}

Image segmentation by means of traditional low-level methods necessitates substantial sum of expert interactive guidance. Numerous boundaries of conventional image processing methods are abridged or even eradicated by 
means of a deformable replica (Glocker, 2011). Deformable replicas are dynamic models (Gaetano , 2015)with respect to the plan of moving a curve or shape underneath the exploited external and internal segments are pointed in Fig 9.

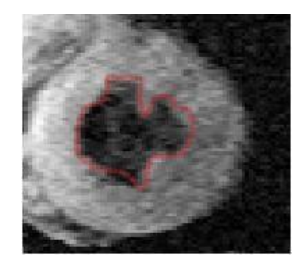

Figure 9. Deformable Model based Image

\subsection{Graph cut method}

Apart from all these techniques given above, graph cut method offers improved outcomes contrast to all (Wei $\mathrm{Hu}, 2015)$. The anticipated scheme computes the lung replicas in a effortless and an effective way (Qian, 2011). An instance of Graph cut method is displayed in fig 10

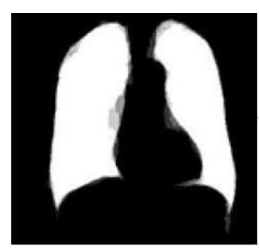

a) Original Image

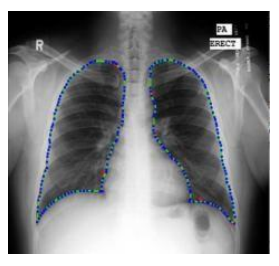

b) output image

Figure 10. Graph Cut Method

\subsection{D joint Markov-Gibbs random field (MGRF) model}

A hybrid approach is expected to segment lung fields from the co-aligned information using a 3D joint Markov-Gibbs random field (MGRF) replica that incorporates three characteristics:

i. the voxel visual manifestation characteristics of the CT image,

ii. the voxels' spatial associations of the CT image, and

iii. To more precisely account for the CT appearance, the voxel appearance replica explains the empirical distribution of image signals by means of a linear combination of discrete Gaussians (LCDG) (Sun, 2012).

The spatial interactions in association with the CT image signals are mock-up by means of a binary pairwise MGRF spatial replica. This improved replica into comprise an adaptive shape prior model that takes into account not merely a voxels location, but as well its intensity data is anticipated to account for the occurrence of pathological tissues. To instruct our segmentation technique to handle pathological fields, the anticipated shape is built from a set of training information sets that are collected from diverse subjects and embrace diverse kinds of pathological tissues. Subsequently, a spatially variant independent random field of area labels of coordinated locations.

Using a support vector machine, the GTV area was segmented. SVM is a machine learning classifier with a high generalization potential, the ability to avoid local smallest pits, and the ability to overcome the curse of dimensionality.

The goal of this research was to feed tumor contours on the basis of the skills and knowledge of radiation oncologists into a training machine, which could be classifiable into GTV and the normal tissue in a test stage by target voxels in an area of interest (ROI). The GTVs were classified by means of a vector support machine that learned three or six voxel-bases in and outside of each tumor region (ground truth). If there was voxel in the GTV area, the indicator for teacher was plus one; the teacher's indicator was minus one if voxel was out of the GTV area. The outside area of the GTV has been separated by a $1 \mathrm{~mm}$ circular kernel six times. At different times, the training voxels were chosen according to the relationship between internal and external voxels, so as to achieve an equal sum of inner and outer voxels. 


\subsubsection{Region Growing}

This procedure removes the history and other extra sections, such as bones, from the lung picture in order to isolate lung tissues and an area of interest (ROI). If the non-assigned pixels that are neighbors with the region tested are close to the region, the region expands (Sluimer, 2006) rapidly, so if the variations between neighboring pixel and region are greater than the threshold, the process is halted. Figure 11 depicts the replica for area development.

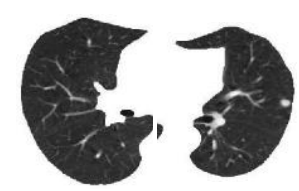

Figure 11. Region growing

Table 1. Five Major Classes Of Ct Lung Segmentation Methods

\begin{tabular}{|c|c|c|c|}
\hline $\begin{array}{c}\text { Lung Segmentation } \\
\text { Class }\end{array}$ & Application & Advantages & Disadvantages \\
\hline Threshold based methods & $\begin{array}{l}\text { Acknowledgment } \\
\text { and } \\
\text { segmentation of } \\
\text { well-defined } \\
\text { standard } \\
\text { structures and } \\
\text { cut off lesions } \\
\text { like tumors, } \\
\text { cavities, and } \\
\text { nodules }\end{array}$ & $\begin{array}{l}\text { Essential, intuitive, } \\
\text { speedy, smallest } \\
\text { Amount expensive } \\
\text { computationally }\end{array}$ & $\begin{array}{l}\text { Sail to contract with } \\
\text { attenuation variations, } \\
\text { fails to classify } \\
\text { pathologic categorization }\end{array}$ \\
\hline $\begin{array}{l}\text { Region-based } \\
\text { methods }\end{array}$ & $\begin{array}{l}\text { Formal structures, } \\
\text { areas with } \\
\text { minimum noise, } \\
\text { minimum } \\
\text { abnormality }\end{array}$ & $\begin{array}{l}\text { Rapid, works well with } \\
\text { more-subtle } \\
\text { attenuation variations }\end{array}$ & $\begin{array}{l}\text { Sails to section regions } \\
\text { with modest to greater } \\
\text { levels of abnormality or } \\
\text { when pathologic } \\
\text { circumstances abuts } \\
\text { adjacent structures }\end{array}$ \\
\hline $\begin{array}{l}\text { Machine learning- based } \\
\text { methods }\end{array}$ & $\begin{array}{l}\text { elineation of } \\
\text { pathologic } \\
\text { circumstances } \\
\text { with signature } \\
\text { textured patterns } \\
\text { like GGO, } \\
\text { consolidation, } \\
\text { and crazy-paving } \\
\text { pattern }\end{array}$ & $\begin{array}{l}\text { Works well to } \\
\text { recognizen on pecific } \\
\text { diffuse pathologic } \\
\text { circumstances, classify } \\
\text { pathologic } \\
\text { classifications like } \\
\text { GGO, consolidation, } \\
\text { and septal line }\end{array}$ & $\begin{array}{l}\text { Computationally cost, no } \\
\text { good divisionamongst } \\
\text { classes of patho- logic } \\
\text { circumstances }\end{array}$ \\
\hline $\begin{array}{l}\text { Neighboring anatomy- } \\
\text { guided methods }\end{array}$ & $\begin{array}{l}\text { Recognition and } \\
\text { classification of } \\
\text { pleural effusions. }\end{array}$ & $\begin{array}{l}\text { Works well in } \\
\text { attenuation-grounded } \\
\text { matrices fail }\end{array}$ & $\begin{array}{l}\text { Computationally } \\
\text { expensive, rigorous } \\
\text { pathologic circumstance } \\
\text { could throw it off (eg, } \\
\text { opacification of complete } \\
\text { hemithorax) }\end{array}$ \\
\hline $\begin{array}{l}\text { Shape-based methods } \\
\text { (atlas- based } \\
\text { and model- based } \\
\text { methods) }\end{array}$ & $\begin{array}{l}\text { Irregular } \\
\text { pathologic } \\
\text { circumstances } \\
\text { that defy } \\
\text { segmentation of } \\
\text { standard anatomy }\end{array}$ & $\begin{array}{l}\text { well-conceived repre- } \\
\text { sentative template } \\
\text { segmentation accuracy } \\
\text { can be high }\end{array}$ & $\begin{array}{l}\text { Representative training } \\
\text { features complex to } \\
\text { training information }\end{array}$ \\
\hline
\end{tabular}




\section{Problem Specification and Future Work}

The rising role of software and image processing in clinical radiology pinpoints the requirements for better awareness amongst radiologists. Certain fields of radiology exploiting computer-aided identification methods for lesion recognition like examination of lung and breast nodules. Nevertheless, the upcoming possibility of computer-aided recognition in radiology is substantial as segmentation procedures persist to progress in regard to the distinction of yield and the proficiency of these techniques in radiologists' working circumstances. In reality, computer-aided detection schemes are not anticipated to substitute radiologists but quite to be complementary to their analytic tasks.

However, to prevail over these restrictions by generate segmentation outcome that embrace the lesion(s) in a three-dimensional description, so that lung pathologic circumstances can be intended as a percentage of the sum lung volume for assessing severity and acquiescent rates of transform in disease with sequential CT examinations. Future works will seek to progress the excellence of segmentation (of lung volumes and disease), increase the effectiveness of these software platforms, and amalgamate the algorithms so that the user interface can faultlessly incorporate with picture archiving and communication system.

\section{Conclusion}

This literary work provides a thorough evaluation of present lung segmentation approaches on images of CT that assist clinicians while selecting instruments to segment the lung area. In five broad divisions, we have divorced the lung field segmentation approaches into a general idea of comparative advantages and disadvantages of each group's techniques. This thesis and the following guidelines would enhance the diagnostic approach by radiologists, thus leading the range and use of computerized segmenting methods for pulmonary diagnosis. This analysis provides a comprehensive overview of segmentation strategies to identify a structure or lesion, to draw out contours across the object edges, and then to draw the structure away from adjacent structures for threedimensional evaluation.

In future, the neural networks with swarm intelligence based segmentation schemes will focus to improve the segmentation accuracy and reduce false error rate. Some other features like shape and texture are used for efficient segmentation analysis.

\section{References}

1. Amutha, A., Wahidabanu, R.S.D. (2012) A Novel Method for Lung Tumor Diagnosis and Segmentation using Level Set- Active Contour Modelling”, European Journal of Scientific Research, 90(2) : pp.175- 187

2. Amutha, A., Wahidabanu, R.S.D. (2012) A Novel Method for Lung Tumor Diagnosis and Segmentation using Level Set- Active Contour Modelling”, European Journal of Scientific Research,.90,.2:175- 187.

3. Aroulanandam VV, Latchoumi TP, Balamurugan K, Yookesh TL. (2020) Improving the Energy Efficiency in Mobile Ad-Hoc Network Using Learning-Based Routing, Revue d'Intelligence Artificielle, Vol 34(3), pp. 337-343, 2020.

4. Balamurugan K. (2020) Metrological changes in surface profile, chip, and temperature on end milling of M2HSS die steel. International Journal of Machining and Machinability of Materials, 22(6):pp. 443-453.

5. Balamurugan K and Uthayakumar M, (2017) Preparation And Machining Studies Of Lapo4 Y2o3 Ceramic Matrix Composite, http://hdl.handle.net/10603/166221.

6. Balamurugan K, Uthayakumar M, Sankar S, Hareesh US, Warrier KG. (2020) Process optimisation and exhibiting correlation in the exploitable variable of AWJM. International Journal of Materials and Product Technology, 61(1), pp.16-33Chen X, Udupa JK, Bagci U, Zhuge Y, Yao J. (2012) Medical image segmentation by combining graph cuts and oriented active appearance models. IEEE Trans Image Process, 21(4):2035-2046.

7. Bhasha, A.C., Balamurugan, K. (2020) End mill studies on Al6061 hybrid composite prepared by ultrasonic-assisted stir casting. Multiscale and Multidiscip. Model. Exp. and Design, https://doi.org/10.1007/s41939-020-00083-1

8. ChinnamahammadBhasha, A., Balamurugan, K. (2020) Studies on Al6061nanohybrid Composites Reinforced with SiO2/3x\% of TiC -a Agro-Waste. Silico,. https://doi.org/10.1007/s12633-020-00758-x

9. Chinnamahammad Bhasha and Balamurugan K, (2020) Multi-objective optimization of high-speed end milling on Al6061/3\% RHA/ 6\% TiC reinforced hybrid composite using 
Taguchi coupled GRA, International Conference on Computational Intelligence for Smart Power System and Sustainable Energy (CISPSSE), Keonjhar, India, 2020, pp. 16, doi: 10.1109/CISPSSE49931.2020.9212295.

10. Chaudhary, A.,. Singh, S. S. (2012)Lung Cancer Detection on CT Images by Using Image Processing," Comput. Sci. (ICCS), 2012 Int. Conf.pp. 142-146.

11. Chinnamahammad bhasha A, and Balamurugan, K. (2020) Fracture analysis of fuselage wing joint developed by aerodynamic structural materials." Materials Today: Proceedings, Vol.38, pp. 2555 2562.

12. Chen, X., Udupa, J.K., Bagci, U., Zhuge, Y., Yao. J.(2012)Medical image segmentation by combining graphcuts and oriented active appearance models. IEEE Transactions on Image Processing, 21(4), 2035-2046, 2012.

13. Chu. C et al., (2013)Multi-organ segmentation based on spatially-divided probabilistic atlas from $3 d$ abdominal ct images," in MICCAI, pp. 165-172.

14. Deepthi T, and Balamurugan K. (2019) Effect of Yttrium (20\%) doping on mechanical properties of rare earth nano lanthanum phosphate (LaPO4) synthesized by aqueous sol-gel process. Ceramics International. 45(15), pp.18229-18235.

15. Diciotti S, Lombardo S, Falchini M, Picozzi G. (2011) Mascalchi M. Automated segmentation refinement of small lung nodules in CT scans by local shape analysis. IEEE Trans Biomed Eng;58(12):3418-3428.

16. Glocker, B., Sotiras, A., Komodakis, N., Paragios, N. (2011) Deformable medical image registration: Setting the state of the art with discrete methods," Annual Review of Biomedical Engineering, 13:219-244, 2011.

17. Garikapati P, Balamurugan K, Latchoumi TP, Malkapuram R. (2020) A Cluster-Profile Comparative Study on Machining AlSi $7 / 63 \%$ of SiC Hybrid Composite Using Agglomerative Hierarchical Clustering and K-Means. Silicon. https://doi.org/10.1007/s12633-020-00447-9

18. Gaetano, R., Masi, G., Poggi, G., Verdoliva, L., Scarpa, G. (2015)"Marker-Controlled WatershedBased Segmentation of Multiresolution Remote Sensing Images IEEE Transactions on Geoscience and Remote Sensing, 53, (6):2987-3004.

19. Hashemi A, Pilevar A H, Rafeh R (2013) Mass Detection in Lung CT Images Using Region Growing Segmentation and Decision Making Based on Fuzzy Inference System and Artificial Neural Network, International Journal of Image, Graphics and Signal Processing (IJIGSP), 6, :16-24.

20. Hari Om Shanker Mishra and Smriti Bhatnagar.(2014) MRI and CT Image Fusion Based on Wavelet Transform, International Journal of Information and Computation Technology. ISSN 0974-2239 4( 1): pp. 47-52

21. Latchoumi TP, Reddy MS, Balamurugan K. (2020) Applied Machine Learning Predictive Analytics to SQL Injection Attack Detection and Prevention. European Journal of Molecular \& Clinical Medicine.;7(02), pp. 3543-3553.

22. Khin Mya Mya Tun, Khaing (2014) Implementation of lung Cancer Nodule Feature Extraction using Digital image Processing, International Journal of Scientific Engineering and Technology Research 3 (9) :2204-2210.

23. Khin Mya Mya Tun, Khaing (2014) Implementation of Lung Cancer Nodule Feature Extraction using Digital Image Processing, International Journal of Scientific Engineering and Technology Research, 3 (9):2204-2210

24. Lassen, B., Rikxoort, E.M., Schmidt, M., et al. (2013)Automatic segmentationof the pulmonary lobes from chest CT scans based on fissures, vessels, and bronchi', IEEE Trans. Med. Imaging, , 32, (2),pp. 210-222

25. Lo, P., Sporring, J., Ashraf, H., Pedersen, J. J., and de Bruijne, M. (2010). Vessel-guided airway tree segmentation: A voxel classification approach. Medical Image Analysis, 14(4):527 - 538.

26. Mansoor A, Bagci U, Xu Z, et al.( 2014) A generic approach to pathological lung segmentation. IEEE Trans Med Imaging;33(12):2293-2310.

27. Mesanovic, N., Grgic, M., Huseinagic, H., Males, M., Skejic, E., Smajlovic, M. (2011)Automatic CT Image Segmentation of the Lungs with Region Growing Algorithm," 18th Int. Conf. Syst. Signals Image Process., pp. 395-400,.

28. Manoj R. Tarambale and Nitin S. Lingayat,(2013)Computer Based Performance Evaluation of Segmentation", International Journal of Bioscience, Biochemistry and Bioinformatics, 3 (6).

29. Pavan MV, Balamurugan K, Balamurugan P. (2020) Compressive test Fractured Surface analysis on PLA-Cu composite filament printed at different FDM conditions. 
InIOP Conference Series: Materials Science and Engineering, Vol. 988,pp. 012019. IOP Publishing.

30. Nunes, É.d.O., Pérez, M.G. (2010) Medical Image Segmentation by Multilevel Thresholding Based on Histogram Difference," in17th International Conference on Systems, Signals and Image Processing.

31. Qian Wang, Jiangping Gong, Enmin Song, and Wei Xiong(2011) Computerized quantification menthod for lung nodule speculation features in CT images", Huazhong Univ.of Sci.\& Tech.(Natural Science Edition.39(7).

32. Sluimer I, Schilham A, Prokop M, van Ginneken B. (2006) Computer analysis of computed tomography scans of the lung: a survey. IEEE Trans Med Imaging,25(4): 385-405.

33. Sun S, Bauer C, Beichel R. (2012) Automated 3-D segmentation of lungs with lung cancer in CT data using a novel robust active shape model approach. IEEE Trans Med Imaging;31(2):449-460.

34. Suzuk, K., (2012) Pixel-based machine learning in medical imaging," Journal of Biomedical Imaging, 2012, pp. 1-18

35. Sunil Kumar, Ashok Kumar (2014)Lung Segmentation Using Region Growing Algorithm”, International Journal of Advanced Research in Computer Science and Software Engineering 4(3), pp. $184-187$

36. Song Y, Cai W, Kim J and Feng D D. (2012)A Multistage Discriminative Model for Tumor and Lymph Node Detection in Thoracic Images, IEEE Transactions on Medical Imaging,31(5): pp 10611075

37. van Rikxoort EM, van Ginneken B. (2013) Automated segmentation of pulmonary structures in thoracic computed tomography scans: a review. Phys Med Biol;58(17): R187-R220.

38. Yao J, Bliton J, Summers RM. (2013) Automatic segmentation and measurement of pleural effusions on CT. IEEE Trans Biomed Eng;60(7):1834-1840.

39. Yrj”o H"ame, Elsa D. Angelini Eric A. Hoffman, R. Graham Barr, Andrew F. Laine (2013)Robust Quantification Of Pulmonary Emphysemawith A Hidden Markov Measure Field Model" IEEE International Conference on Biomedical Imaging:.

40. Wei Hu, Deihui Xiang, Bin Xiang, lirong Wang, Ivica Kopriva, Macgregor, J. H. (2015) Random Walk and Graph Cut for Co-Segmentation of Lung Tumor on PET-CT images," IEEE Trans. On image. processing.24(12): 5854-5866.

41. Wei, Q., Hu, Y. "A Hybrid Approach to Segmentation of Diseased Lung Lobes(2014) IEEE journal of biomedical and health informatics., 18, ( 5): 1696-1706. 